\title{
Crocker and Hartzell's disease - Report of two cases with literature review
}

\author{
Sowmya GV ${ }^{1 *}$, Suveet Mathur ${ }^{2}$ \\ ${ }^{\mathbf{1}}$ Reader, ${ }^{2}$ Private Clinician, ${ }^{\mathbf{1}}$ Dept. of Oral Medicine and Radiology, ${ }^{\mathbf{2}}$ Dept. of Oral \& Maxillofacial Surgery, ${ }^{\mathbf{1}}$ Institute of Dental Sciences, \\ Bareilly, Uttar Pradesh, ${ }^{2}$ Pain Off Dental Care, Jodhpur, Rajasthan, India \\ *Corresponding Author: Sowmya GV \\ Email: tamannamyangel@gmail.com
}

\begin{abstract}
Pregnancy tumor is a tissue overgrowth which usually advances as a reaction to irritation and trauma that is typically localized. Nevertheless gingiva is supposed to be the dominant site, it also can occur on tongue, lip, and buccal mucosa. Pregnancy does not form the basis for the ailment, but changed tissues metabolic rate in pregnancy stresses the retort to the native irritants, thereby generating gingival overgrowths. It is commonly seen in first trimester of gestation. The present article reports two cases with pregnancy tumor in relation to anterior mandible.
\end{abstract}

Keywords: Inflammatory hyperplasia, Pyogenic granuloma, Pregnancy, Gingiva.

\section{Introduction}

The hormonal whims occurring in pregnancy, tips to changes in oral flora building the oral cavity more vulnerable to oral inflammations together with infections. ${ }^{1}$ Well-timed referral by the gynaecologist to the dentist can abate the oral health concerns related to pregnancy. ${ }^{2}$

Pregnancy has been seen to upsurge liability to gingival inflammation. ${ }^{3}$ Pregnancy tumor, nothing but a pyogenic granuloma, that ensues during pregnancy and is said to be non-neoplastic in nature. ${ }^{4}$ In the main, Poncet and Dor were to designate pyogenic granuloma in 1897 and termed it Botryomycosis Hominis. Confusion prevails about the present name, which is believed to be given by Crocker and Hartzell in 1903 \& 1904 respectively, hence goes with the synonym of Crocker and Hartzell's disease. ${ }^{5}$

It is also known otherwise as Granuloma pyogenicum, Granulation tissue-type hemangioma, Granuloma gravidarum, Lobular capillary hemangioma, Pregnancy tumor, Granuloma telangiectacticum, Granuloma pyogenicum, Granuloma pediculatum benignum and Benign vascular tumor. ${ }^{6,7}$

The prevalence is expected to be $5 \%$ of all pregnancies. ${ }^{8}$ They are principally found on the gingiva with incidence ranging $0.2-9.6 \%$ during gravidity. They commonly tend to appear after first trimester, grow swiftly, and classically regress after parturition. ${ }^{9}$ Various authors demonstrated that $55 \%$ of overgrowths involved the maxilla, and $83 \%$ arose in the gingiva. ${ }^{10}$

Various etiological factors that trigger the periodontal inflammation can be chronic irritation, hormonal factors and various drugs. This quickly emergent tumor is found predominantly appear during 2 nd or 3rd month of gestation. ${ }^{11}$ Although its reversion typically occurs after parturition, its interference with the normal function might make its excision inevitable. ${ }^{7}$ Clinically, it characterizes a tumor-like growth, reddish-reddish pink reliant on its vascularity, propensity to bleed with most common location being the interdental papilla of maxillary anterior teeth followed by tongue, lip, palate, and oral mucosa..$^{5,11}$

The documented treatment modalities include complete surgical excision, radiosurgery, cryosurgery, laser surgery, and intralesional injections using ethanol, sodium tetra decylsulfate \& monoethanolamineoleate or corticosteroid. ${ }^{4,12}$ If not completely removed, it has propensity to recur. Whereas, it has a good prognosis. ${ }^{8}$

\section{Case 1}

A 26-year-old female reported to the Outpatient Department of Institute of Dental Sciences, Bareilly, UP with a chief complaint of overgrowth in the lower front tooth region of the oral cavity since one month. There was no pain allied with the lesion. Patient gave a history of her experience of bleeding on brushing with associated difficulty in mastication and speech. Medical history revealed her $6^{\text {th }}$ month pergnancy. Intraoral examination revealed a soft tissue overgrowth in the anterior mandible wrt $32 \& 33$. It was pale pink in color, oval in shape measuring approximately 2 inches $\times 2.5$ inches size. It was with pedunculated base and smooth surface involving the interdental gingiva between 32 $\& 33$ region. On palpation it was soft to firm in consistency, bleeding on palpation was present without ulceration (Fig. 1a). Intra-oral periapical radiograph (IOPA) revealed mild intercrestal bone loss irt $32 \& 33$ with spacing. (Fig. 1b)

The patient was unable to maintain oral hygiene in this area, as of gingival overgrowth, rest of the oral cavity presented normal gingiva with acceptable oral hygiene. A provisional diagnosis of pregnancy tumor was made. The differential diagnosis included was irritational fibroma \& peripheral giant cell granuloma.

As of her pregnancy, a non-invasive excisional biopsy procedure was done with sutures placed under local anaesthesia (Fig. 1c) and sent for histopathological evaluation which confirmed the pregnancy tumor. (Fig. 1d) After Clinico-pathological connexion, a final diagnosis of pregnancy tumor was agreed. 


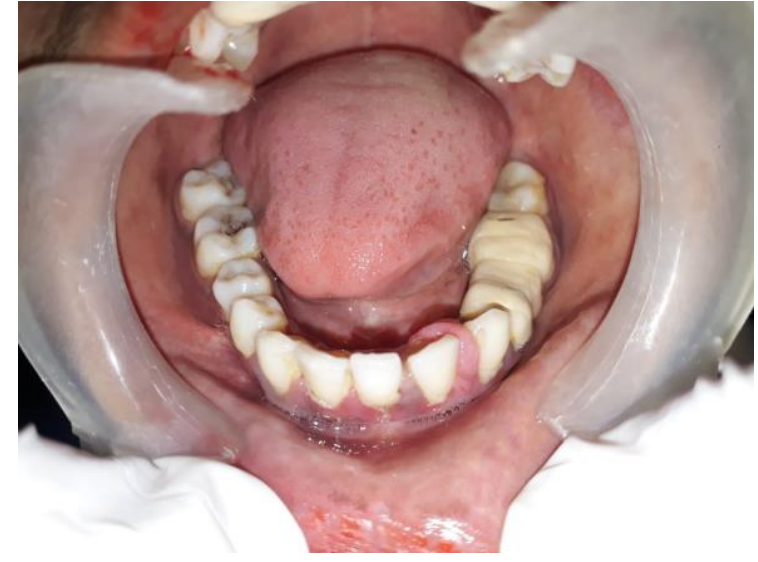

Fig. 1a: Clinical photograph showing overgrowth in the anterior mandible irt 32, 33

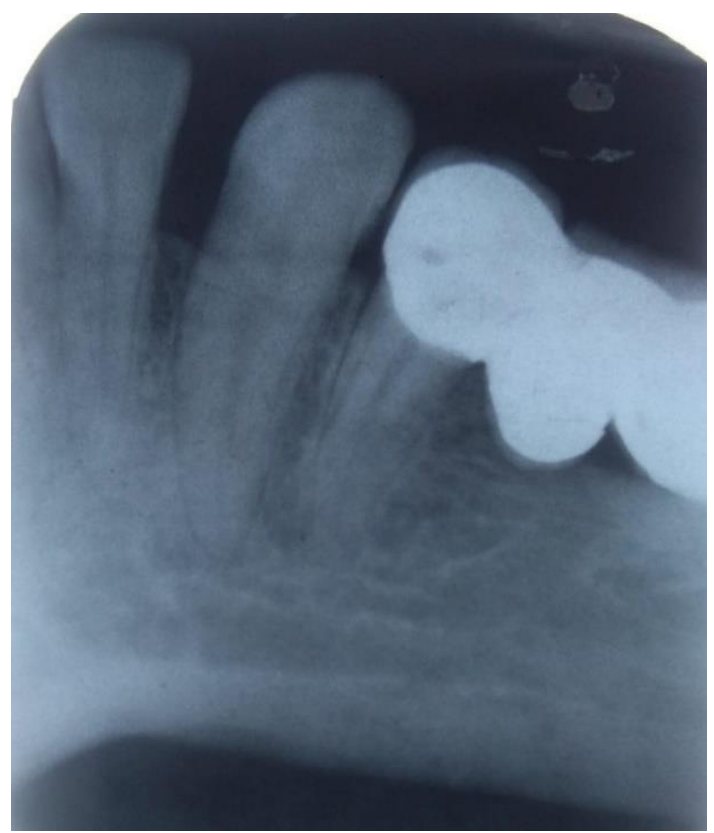

Fig. 1b: Intra oral Periapical radiograph of case 1 showing intercrestal bone loss between 32, 33 with spacing

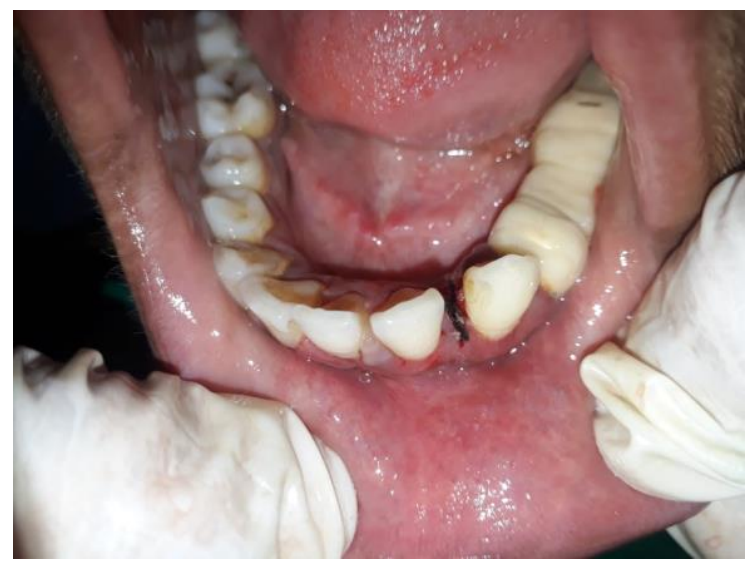

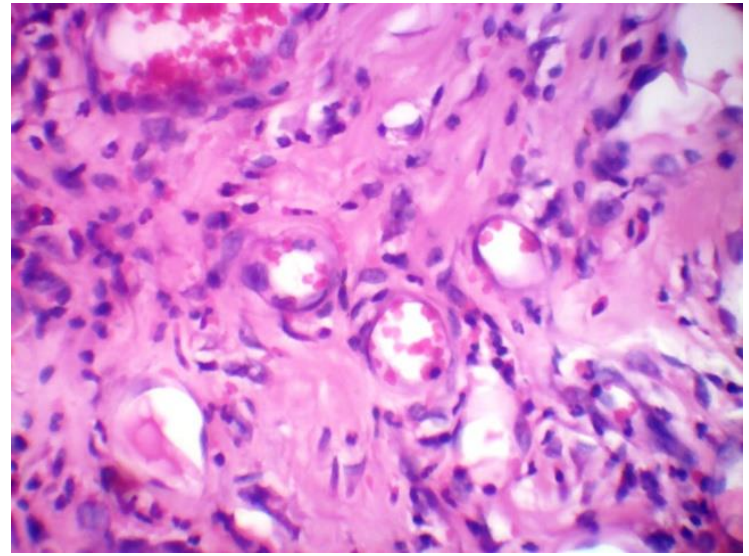

Fig. 1d: Photomicrograph of case 1 showing reveals numerous vascular channels in a background of fibrous connective tissue. A mixed inflammatory cell infiltrate is scattered throughout the stroma

\section{Case 2}

A 24-year-old female reported to the private clinician with an overgrowth of gums in the front region of lower jaw since 25 days. It was initially small in size which had grown to the size of the present day. The patient also complained of bleeding on brushing and while mastication. It was found that the patient was $5^{\text {th }}$ month pregnant. Intra oral examination, revealed a $3 \mathrm{~cm} \times 4 \mathrm{~cm}$ pedunculated nodular mass having reddish pink color with bleeding tendency irt mandibular anterior region (Fig. 2a). The mass was present wrt 31 \& 41 region covering both the labial and lingual area (Fig. 2b). On palpation, it was soft to firm in consistency with bleeding was observed without ulceration. The origin of the growth was observed from the interdental space between the mandibular central incisors with difficulty in maintaining this area clean. Intraoral periapical radiograph revealed intercrestal with mild horizontal bone loss wrt 31 \& 41. (Fig. 2c) A provisional diagnosis of pregnancy tumor was made with considering peripheral giant cell granuloma as differential diagnosis. Later, considering $2^{\text {nd }}$ trimester as safe period, complete excisional biopsy was done using diode laser. The excised gingival overgrowth was sent for histopathological examination. Histopathology revealed the overlying epithelium is stratified squamous parakeratinized type. Connective tissue stroma comprised of dense collagen fibres with predominantly plump shaped fibroblasts. Abundant endothelial lined blood vessels with RBCs and extravasated RBCs are evident. Diffuse chronic inflammatory infiltrate predominantly comprised of lymphocytes, few plasma cells and mononuclear cells was evident. The overall histopathologic features were consistent \& confirming the clinical diagnosis of pregnancy tumor. (Fig. 2d)

Fig. 1c: Sutures placed after biopsy done 


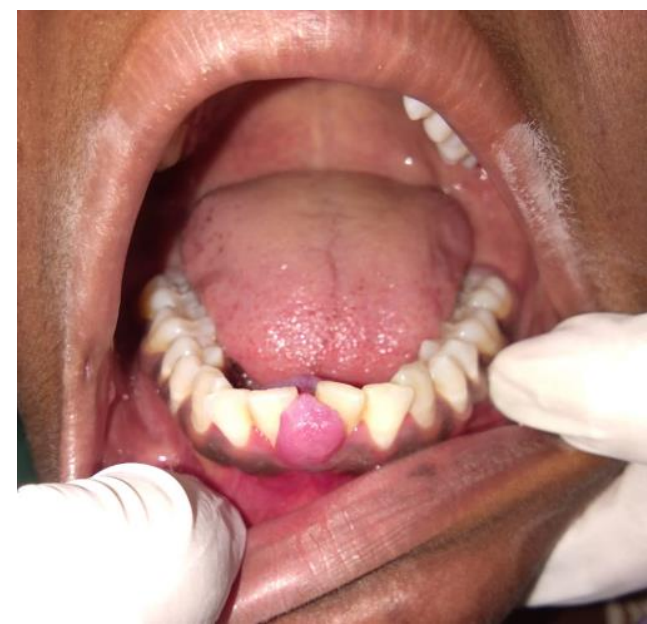

Fig. 2a: Clinical photograph showing a pedunculated gingival overgrowth in anterior mandible between 31,41

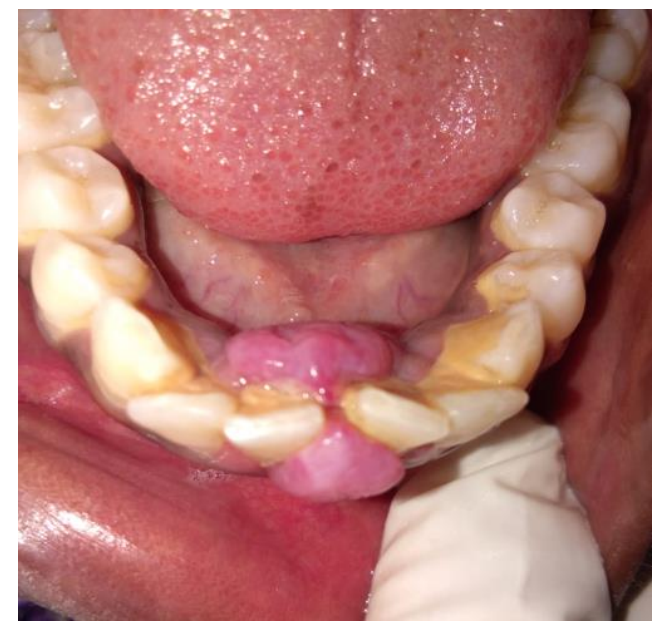

Fig. 2b: The mass was present wrt $31 \& 41$ region covering both the labial and lingual area

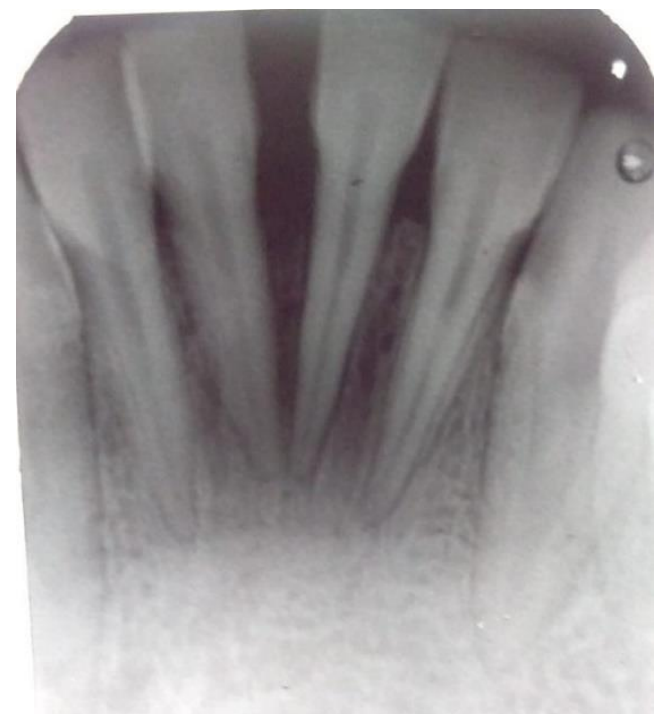

Fig. 2c: Intra oral Periapical radiograph showing mild horizontal bone loss wrt $31 \& 41$

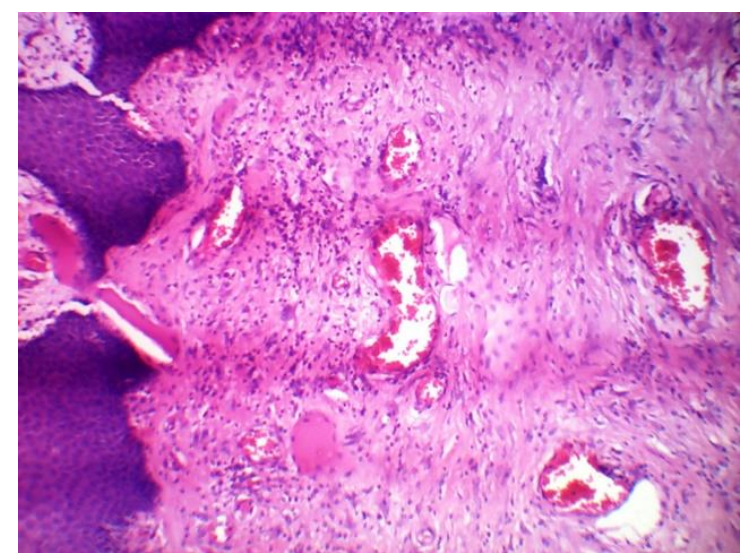

Fig. 2d: Photomicrograph of showing overlying epithelium is stratified squamous parakeratinized type. Connective tissue stroma comprised of dense collagen fibres with predominantly plump shaped fibroblasts. Numerous endothelial lined blood vessels with RBCs and extravasated RBCs were evident.

\section{Discussion}

The pregnancy tumor is nothing but pyogenic granuloma affecting females usually in 3rd-9th month of their pregnancy. ${ }^{13}$ Pyogenic granuloma arises in its anatomic location of gingiva in $5 \%$ of pregnancies. ${ }^{14,15}$

In 1946 Ziskin and Ness collectively gave a clinical classification of pregnancy gingivitis as follows: ${ }^{7,16}$ (Table 1)

Table 1: Ziskin and Ness's clinical classification of pregnancy gingivitis

\begin{tabular}{|l|l|}
\hline Class I & $\begin{array}{l}\text { Characterized by bleeding gingiva with more } \\
\text { or less no other manifestations }\end{array}$ \\
\hline Class II & $\begin{array}{l}\text { Characterized by changes in interdental } \\
\text { papilla-edema and swelling with exhibits a } \\
\text { tendency to recur. Subsequent blunting of } \\
\text { interdental papilla }\end{array}$ \\
\hline Class III & $\begin{array}{l}\text { Characterized by the involvement of the free } \\
\text { gingival margin, which takes on the color and } \\
\text { general appearance of a raspberry }\end{array}$ \\
\hline Class IV & $\begin{array}{l}\text { Generalized hypertrophic gingivitis of } \\
\text { pregnancy }\end{array}$ \\
\hline Class V & The pregnancy tumor \\
\hline
\end{tabular}

Clinical presentation include a tumor-like growth, reddish-reddish pink-purplish red depending on the vascularity of the lesion with propensity to bleed. ${ }^{11,17}$ Intraorally, maxillary anterior gingiva is the most common site than the mandibular gingiva and the labial gingiva is involved more than the lingual gingiva. Literature says that lesions affecting both the labial and lingual gingivae are considered rare. ${ }^{18}$ Both the cases in the present report were seen in the anterior part of mandible which has the $2^{\text {nd }}$ highest rate of occurrence of pregnancy tumor followed by anterior maxilla comprising both facial and lingual gingivae which is said to be rare. Whereas Sakshi Sareen in $2015,{ }^{19}$ Saravana in 2009 after his review on 137 cases, demonstrated that $55 \%$ of pyogenic lesions involved the maxilla, ${ }^{20}$ and $83 \%$ occurred in 
the gingiva and U. Ar Unmozhi described a case huge pregnancy tumor of tip of tongue in 2016 in his case report. ${ }^{5}$

In oral cavity, it is usually seen affecting the keratinized mucosa. Numerous aetiologies postulated to play a role include trauma, hormonal influences, viral oncogenes, underlying minute arteriovenous distortion, the production of angiogenic growth factor and cytogenic aberrations. ${ }^{8,21}$ Whereas, deviant tooth development, occlusal interferences, immunosuppressive medicines such as cyclosporine and incorrect choice of healing cap for implants are some of the other activating factors for pyogenic granulomas. ${ }^{22}$ The basic mechanisms involved in the development of pyogenic granuloma deceits in the endocrinal changes leading to alterations in blood and lymph microvasculature of skin and mucosa. ${ }^{13}$

Ojanotko-Harri et al. in 1991, emphasized progesterone as the source in pregnancy gingivitis and associated tumor. ${ }^{23}$ In our case hormonal imbalance due to pregnancy was supposed to be the aetiological factor. The etiologic cause by which feminine sex steroid hormones may affect the periodontium of women during pregnancy, are diverse and differ from those usually associated with plaque-induced gingivitis. ${ }^{24}$ Human gingiva including various receptors for estrogen and progesterone together with amplified plasma levels result in an increased accretion of these hormones in gingival tissues. These surging of estrogen and progesterone levels in circulating blood during pregnancy exerts proliferative effect on the endothelium..$^{25}$ O'Neil in 1979 conveyed that pregnancy can lead to reduction in motherly \& protective T-lymphocytes, diminishing the mother's immunity. Further, this acts as a co-factor in exuberating the tissue response to the plaque microorganisms. ${ }^{11}$ In our cases reports, hormonal imbalance owing to pregnancy was supposed to be the aetiological factor.

On radiographs, there is no frank associated changes are deceptive in granuloma pyogenicum. In erratic instances "localized resorption of alveolar bone" of chronic tumors of gingiva which was noted by Angelopoulos. Differential diagnosis includes Fibroma, Peripheral giant cell granuloma, Vascular lesion like hemangioma, Inflammatory gingival hyperplasia. ${ }^{6}$

Histologically, the lesion is composed mainly of immature vascular granulation tissue. There are two histologic types namely - lobular capillary hemangioma and non-nodular capillary hemangioma, which show different histologic features. Microscopically, this reactive gingival hyperplasia is filled with unformed fibroblastic connective tissue, proliferative blood vessels and inflammatory cells. ${ }^{26}$ Complications consist of hemorrhage, infection, and ulceration. Nevertheless, no malignant potential is reported in the literature till date. ${ }^{6}$

Management of pregnancy granuloma rests on the evaluations of the symptoms. If the lesion is slight, painless with no bleeding, clinical observation and follow- up are suggested. During pregnancy, surgery is recommended only if bleeding or pain from the lesion hinders daily activities, or after delivery, if the lesion has not reverted completely. ${ }^{8}$ Additional measures like cryosurgery, laser therapy.
Recently, cases managed with sclerotherapy using sodium tetra decyl sulfate and monoethanolamineoleate, have also been documented. ${ }^{18}$ Many lasers like Diode laser, $\mathrm{CO} 2$ (Carbon Dioxide Laser), Nd:YAG (Neodymium-Doped Yttrium, Aluminium Garnet) and Er:YAG (Erbium-Doped Yttrium Aluminum Garnet) have allegedly been successful in handling mucosal pyogenic granulomas. The probability of recurrence of the lesion looks that the use of laser will lessen this recurrence rate. ${ }^{21}$

\section{Conclusion}

Oral health maintenance in pregnancy is often dodged and misread by both dentists and patients. Pregnancy tumor is a reactive haemorrhagic swelling due to irritation or physical trauma from calculus or cervical restorations together with contribution by hormonal factors usually affecting the gingiva, seen during gestation. It typically progresses after the third month of pregnancy and may attain a huge size, thus, necessitating its surgical elimination. So, clinicians should have thorough knowledge regarding its clinical presentation, with proper diagnosis, prevention and management that is favourable for both the patient and fetal care.

\section{Acknowledgements}

None.

\section{Conflict of interest}

None declared.

\section{Information consent}

Obtained.

\section{References}

1. Laine, Anneli M. Effect of pregnancy on periodontal and dental health. Acta Odontol Scand 2002;60:257-64.

2. Naseem M, Khurshid Z, Khan HA, Niazi F, Zohaib S, Zafar MS: Oral health challenges in pregnant women:

Recommendations for dental care professionals. Saudi J Dent Res 2015;7:138-46.

3. Wu M, Chen S-W, Jiang S-Y. Relationship between Gingival Inflammation and Pregnancy. Mediators Inflamm 2015, 2015:1-11.

4. Gondivkar S, Gadbail A, Chole R. Oral pregnancy tumor. Contemp Clin Dent 2010;1:190-2.

5. Arunmozhi U, Priya RS, Kadhiresan R, Sujatha G, Shamsudeen-SS SM: A large pregnancy tumor of tongue-a case report. J Clin Diagn Res 2016;10:ZD10-ZD2.

6. Nagaraj T, Irugu K, Okade D, Saxena S. Extragingival pyogenic granuloma on the tongue: A rare case report and review of literature. J Med Radiol Pathol Surg 2017;4:10-3.

7. Phore S, Panchal R. Pregnancy tumor: A rare case report in mandibular anteriors. BLDE Univ J Health Sci 2017;2:112-4.

8. Esmaeil N, Sharmila B, Sangeeta M, Rahul K: A case report of pregnancy tumor and its management using the diode laser. $J$ Dent Lasers 2012;6:68-71.

9. Rockafellow A, Florin W, Philipone E, Koslovsky D. Pregnancy Tumor in a 31-Year-Old Female with a Facial PortWine Stain. Case Rep Dent 2015, 2015:472605-11.

10. Sills E, Zegarelli D, Hoschander M, Strider W: Clinical diagnosis and management of hormonally responsive oral pregnancy tumor (pyogenic granuloma). J Reprod Med 1996;41:467-70. 
11. Nisha S, Shivamallu A, Hedge U. Oral pregnancy tumor. $J$ Dent Allied Sci 2018;7:47-50.

12. Sun W-L, Lei L-H, Chen L-L, Yu Z-S, Zhou J-W. Multiple gingival pregnancy tumors with rapid growth. Journal of Dental Sciences 2014, 9:289-93.

13. Jafarzadeh H, Sanatkhani M, Mohtasham N. Oral pyogenic granuloma: a review. J Oral Sci 2006;48:167-75.

14. Tabatabaei N, ES, Taheri J, Azimi S. Frequency of Gingival Pregnancy Tumor in Iran (Confirmed by Biopsy). J Int Oral Health 2014;6:72-6.

15. Kerr, A D: Granuloma pyogenicum. Oral Surg Oral Med Oral Pathol 1951;4:158-76.

16. Stark M, Zarka F: Gingival tumors of pregnancy; review of pregnancy tumors and a report of two cases. Obstet Gynecol 1956;8:597-600.

17. Kashyap B, Reddy PS, P N. Reactive lesions of oral cavity: A survey of 100 cases in Eluru, West Godavari district. Contemp Clin Dent 2012;3:294-7.

18. Reddy NR, Kumar PM, Selvi T, Nalini HE. Management of Recurrent Post ${ }^{-}$partum Pregnancy Tumor with Localized Chronic Periodontitis. Int J Prev Med 2014;5:643-7.

19. Sareen S, Baburaj M, Pimpale S. Pregnancy tumor: case report of two cases. IJSS Case Rep Rev 2015;2:14-8.

20. Saravana G: Oral pyogenic granuloma: A review of 137 cases. Br J Oral Maxillofac Surg 2009;47:318-9.

21. Fekrazad R, Nokhbatolfoghahaei H, Khoei F, Kalhori K. Pyogenic Granuloma: Surgical Treatment with Er: YAG Laser. J Lasers Med Sci 2014;5:199-205.

22. Gomes S, Shakir Q, Thaker P, Tavadia J. Pyogenic granuloma of the gingiva: A misnomer?-A case report and review of literature. J Indian Soc Periodontol 2013;17:514-9.

23. Ojanotko-Harri A, Harri M, Hurttia H, Sewón L. Altered tissue metabolism of progesterone in pregnancy gingivitis and granuloma. J Clin Periodol 1991;18:262-6.

24. Markou E, Eleana B, Lazaros T, Antonios K. The Influence of Sex Steroid Hormones on Gingiva of Women. Open Dent $J$ 2009;3:114-9.

25. Jafri Z, Bhardwaj A, Sawai M, Sultan N. Influence of female sex hormones on periodontium: A case series. J Nat Sci Biol Med 2015, 6:S146-S9.

26. Marla V, Shrestha A, Goel K, Shrestha S. The Histopathological Spectrum of Pyogenic Granuloma: A Case Series. Case reports in dentistry. 2016:1323798-804.

How to cite this article: Sowmya GV, Suveet Mathur. Crocker and Hartzell's disease - Report of two cases with literature review. J Oral Med, Oral Surg, Oral Pathol, Oral Radiol 2019;5(3):88-92. 